\title{
Continuous curvilinear (circular) capsulorhexis and planned extracapsular cataract extraction - are they compatible?
}

\author{
Milind Pande
}

Abstract

The successful use of continuous curvilinear (circular) capsulorhexis (CCC) in planned extracapsular cataract extraction (ECCE) is dependent on the feasibility of safe nuclear delivery through the smaller anterior capsular opening. Experimental evidence supports the proposition that the anterior capsular rim can stretch without tearing to dimensions which allow for safe nucleus delivery. Clinical reports of accidental intracapsular expression during nucleus delivery in CCC seemingly contradict the experimental evidence. This paper examines this apparent contradiction and presents clinical evidence in the form of a series of 210 cases of planned ECCE with CCC. The technique used and a detailed analysis of the operative complications are presented. The results indicate that using a modified technique for nucleus delivery, CCC and planned ECCE are fully compatible techniques.

(Br F Ophthalmol 1993; 77: 152-157)

Continuous curvilinear (circular) capsulorhexis (CCC) provides the cataract surgean with a completely intact capsular bag for lens implanta-

Department of Ophthalmology, Hull Royal Infirmary $M$ Pande

Correspondence to: Mr M Pande, Iris Fund Research Fellow, Medical Eye Unit, Department of Ophthalmology, St Thomas's Hospital, London SEI 7EH. Accepted for publication 3 November 1992

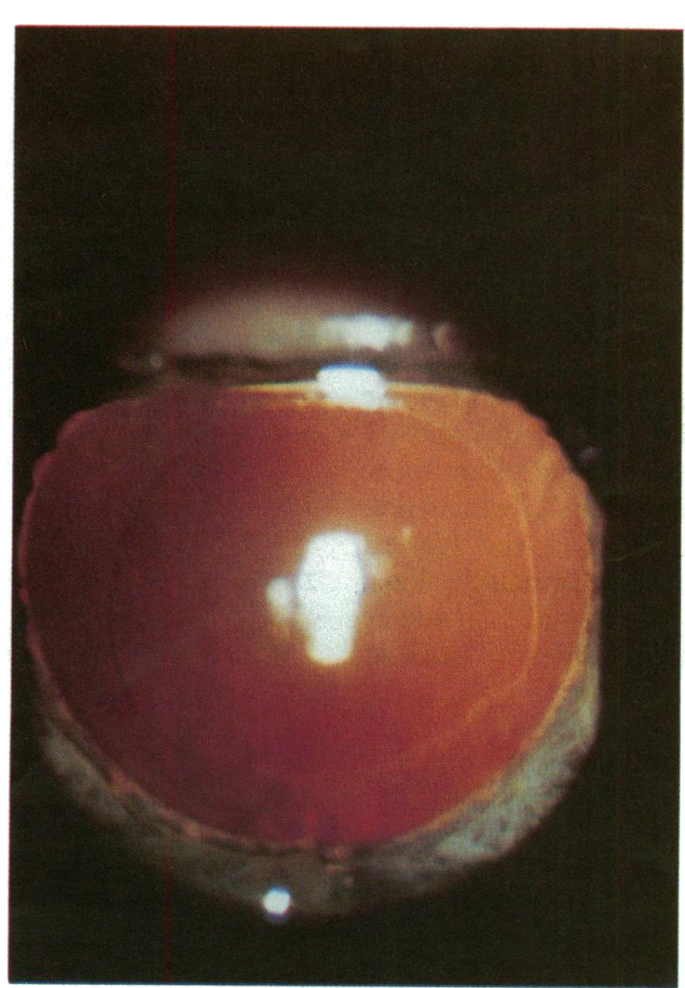
Postoperative photograph showing the continuous anterior capsular rim in front of the implant.
Figure 1 Clinically verifiable 'in the bag' fixation.

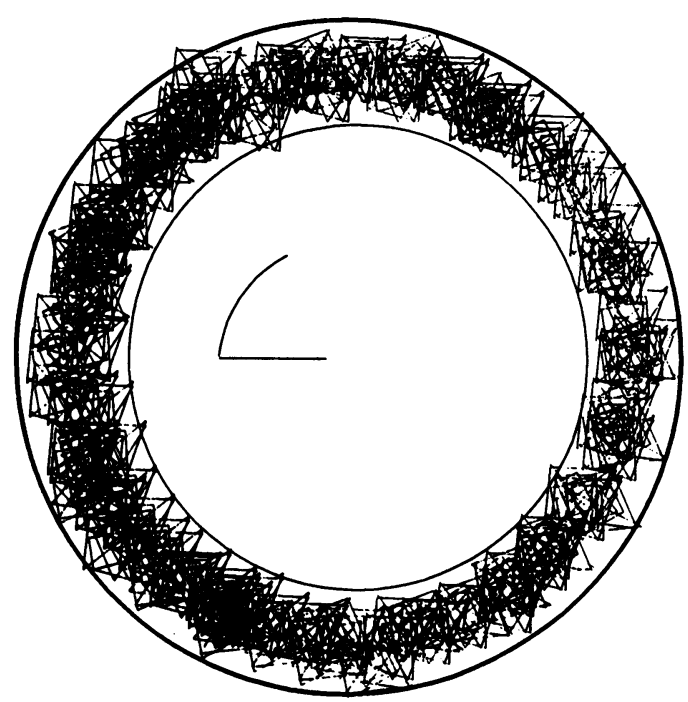

Figure 2 The initial radial and circumferential cuts in the capsule to create an anterior capsular flap.

tion. It is the only method of anterior capsulotomy which allows a complete operative and postoperative (Fig 1) visual verification of 'in the bag' placement of an implant; as the anterior capsular rim can be visualised for 360 degrees. ${ }^{1}$ The use of this technique in planned extracapsular cataract extraction (ECCE) has been limited owing to the singular problem of delivering a larger nucleus through the smaller anterior capsular rim. Clinical reports ${ }^{2-4}$ of accidental intracapsular expression with the use of $\mathrm{CCC}$ in

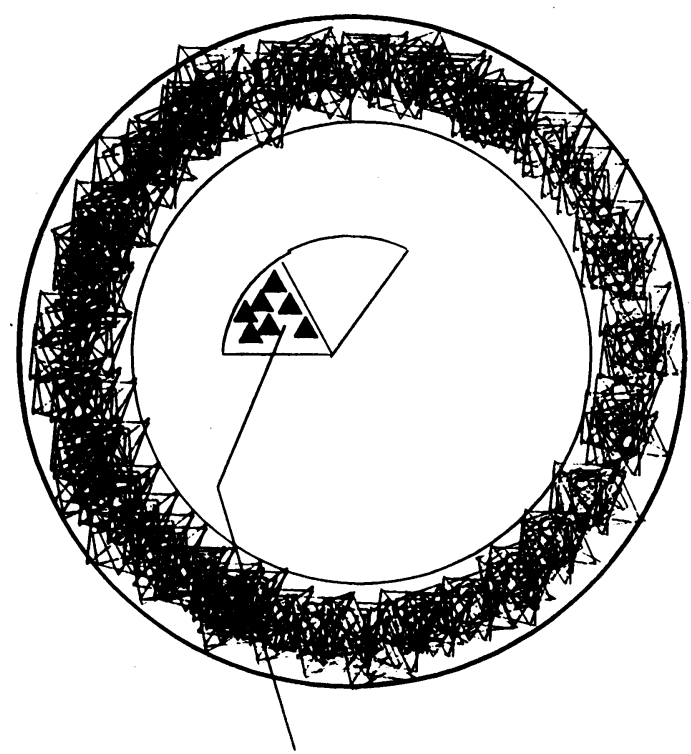

Figure 3 Injection of viscoelastic to raise the capsular flap created as shown in Figure 2. 

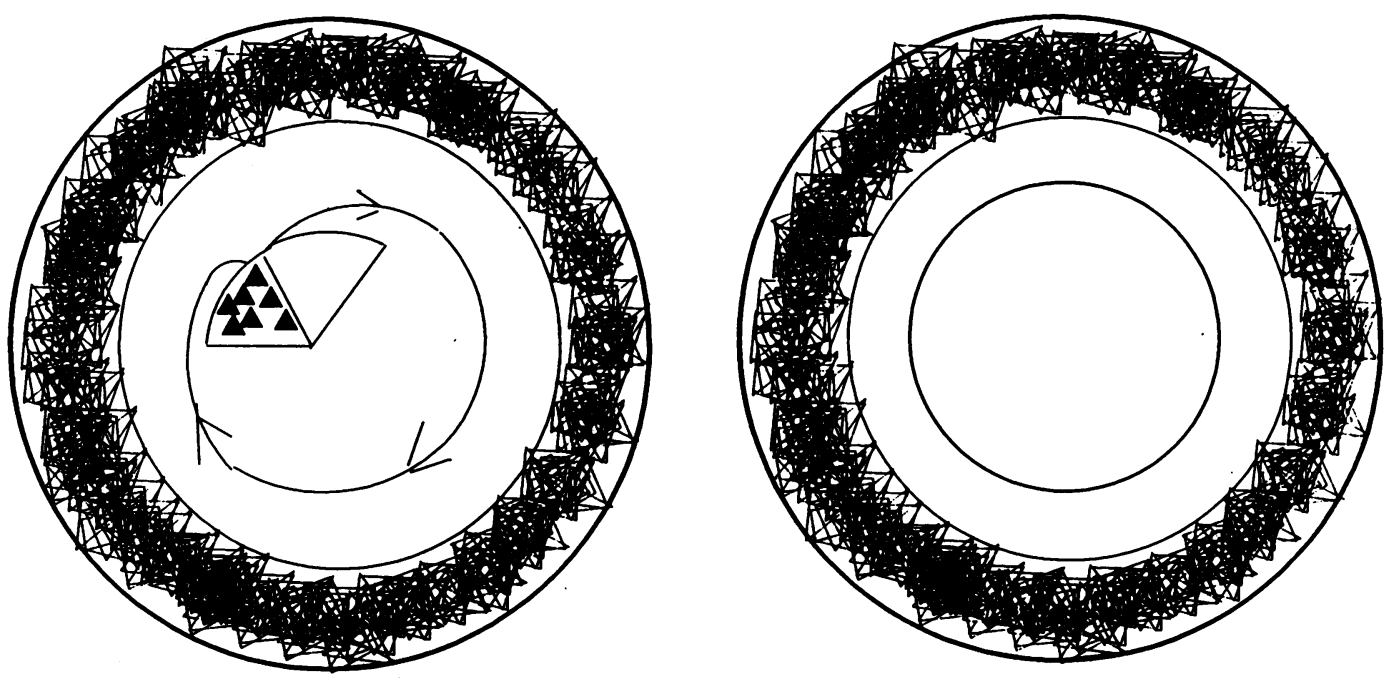

Figure 4 The capsular flap is grasped with a forceps and torn spirally to end beyond the starting point to achieve a smooth edged continuous curvilinear opening.

ECCE have highlighted this problem further. This paper presents clinical evidence in the form of a series of 210 cases who had CCC and planned ECCE.

\section{Patients and methods}

Two hundred and ten eyes of 207 patients operated on by me consecutively from 1 February 1990 to 31 July 1991 at the Hull Royal Infirmary were included. All patients were routine waiting list admissions. Patients with mature or hypermature cataracts or small pupils were excluded during the period from 1 February 1990 to 1 August 1990. The mean age was 72.35 years with a range of 39 to 93 years. There were 97 male and 110 female patients. The follow up ranged from 3-18 months with a mean of $8 \cdot 7$ months.

A specially designed computer database was used to store the preoperative, operative, and postoperative data on all cases. The preoperative data included the visual acuity, type of cataract, and pupillary synechiae. The operative data included all complications, their management, the step at which complications occurred, and the site and size of anterior and posterior capsular tears. The postoperative data included the duration of follow up and the final visual acuity.

All cases underwent extracapsular cataract surgery and implantation with continuous CCC as in the technique described below.

\section{TECHNIQUE}

Pupillary dilatation was achieved by instilling cyclopentolate $1 \%$ and phenylephrine $2 \cdot 5 \%$ drops three times in the hour before surgery. General anaesthesia was used in 190 cases while retrobulbar anaesthesia was given in 20 cases.

A 120 degree corneal section was made and the anterior chamber filled with viscoelastic to stretch the anterior capsule. Continuous CCC was performed. The steps included the creation of a capsular flap (Fig 2) by making a radial and a circumferential cut in the anterior capsule.
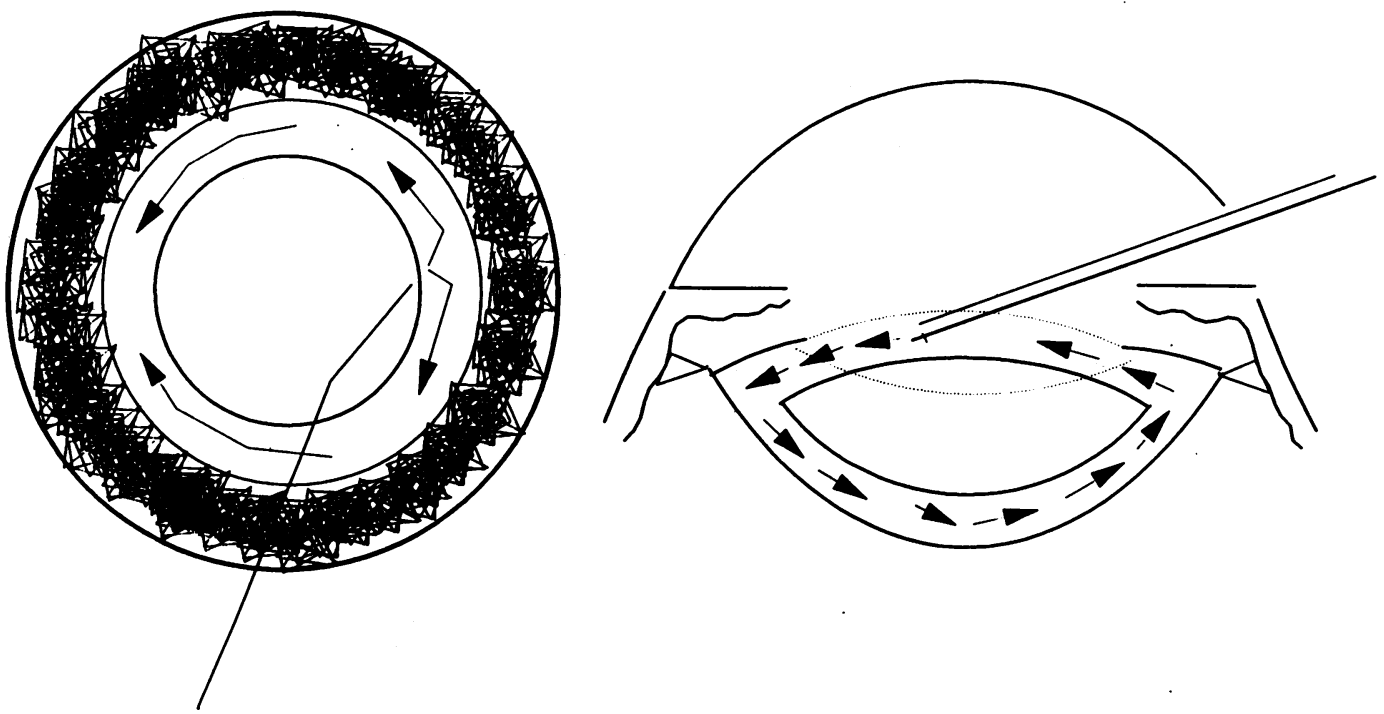

Figure 5 Global hydrodissection. Sectional and top views of the eye. The fluid injection dissects the nucleus free of its cortical attachments along all its surfaces (global). This frees the nucleus completely so as to be able to move independently of the capsular bag. 

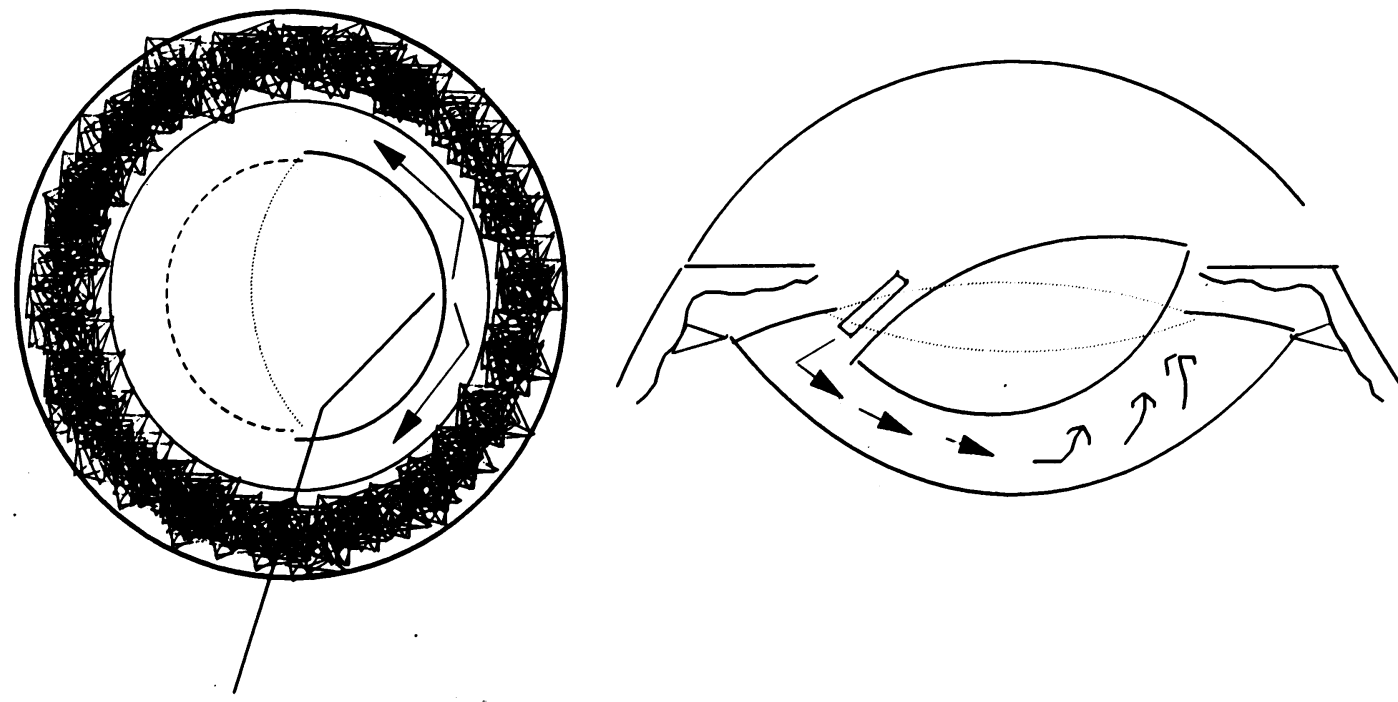

Figure 6. Sectional and top views of the eye. With the nucleus free of its cortical attachments the cannula is placed at the 9 o'clock position and irrigating solution injected into the capsular bag. This raises the fluid pressure in the capsular bag which in turn lifts the 3 o'clock nuclear pole out of the capsular bag.

Viscoelastic was injected under the capsular flap to elevate it (Fig 3). The capsular flap was then torn around with a forceps in a spiral fashion to achieve a curvilinear (circular) anterior capsular opening (Fig 4). The capsulotomy was restricted to a size of 5.5-6 mm so as not to encroach on the zonular insertion into the anterior capsule. No relieving incisions were made.

Nucleus delivery was achieved by the following method. A Rycroft cannula was used to inject irrigating fluid under the anterior capsule in all four quadrants (Fig 5). The fluid dissection was visually monitored to confirm that it had freed the nucleus of all its cortical attachments. The cortical attachments had to be freed not just along the posterior aspect of the nucleus but also along the anterior surface under the residual anterior capsular collar. Thus the nucleus was hydrodissected globally - that is, along all its surfaces. Once the nucleus was completely freed of its cortical attachments, a further injection of irrigating fluid was carried out with the cannula under the anterior capsule at the 9 o'clock position. This fluid injection into the capsular bag caused a pressure build up. As the nucleus is free of cortical attachments this fluid pressure lifted the nuclear pole opposite to the fluid injection area out of the capsular bag as shown in Fig 6 (fluid injected at 9 o'clock the 3 o'clock pole would lift out of the capsular bag).

The cannula was now positioned under the nuclear pole which had lifted out of the capsular bag (3 o'clock pole) and the nucleus is rotated towards the 12 o'clock position (Fig 7). This rotation when completed would have most of the nucleus out of the capsular bag and into the anterior chamber. At this stage slight limbal pressure at the 12 o'clock position delivered the whole nucleus out of the eye.

Cortical aspiration (Fig 8) was carried out using a coaxial irrigation aspiration cannula. The capsular bag was inflated with viscoelastic and a
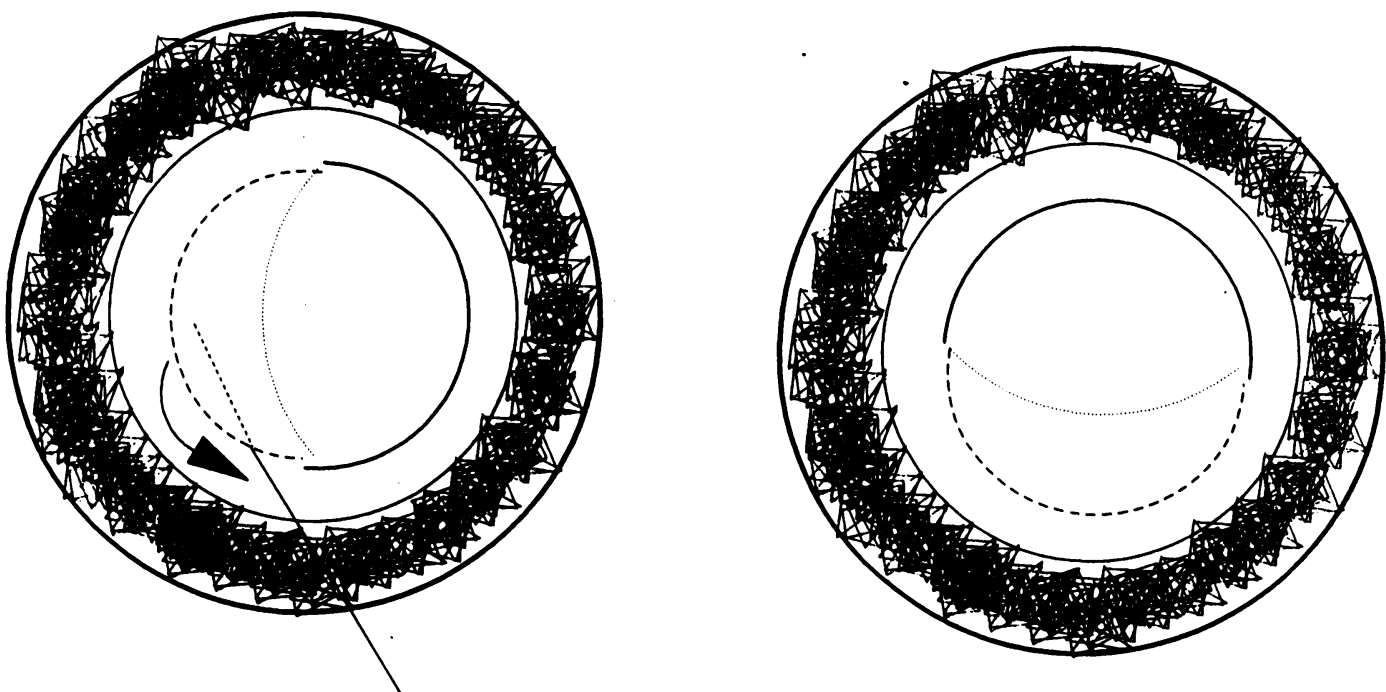

Figure 7 The cannula is then positioned at the $3 o^{\prime}$ clock position under the nuclear pole (now out of the capsular bag) and the pole is rotated to the $12 o^{\prime}$ clock position. this step delivers most of the nucleus out of the capsular bag. Slight limbal pressure is applied to deliver the nucleus out of the eye. 


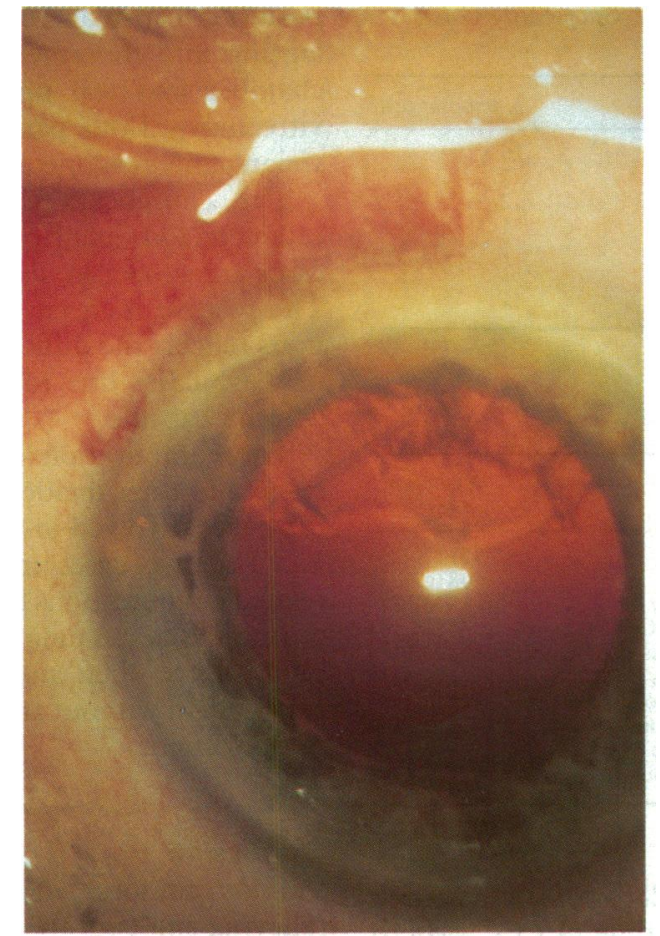

Figure 8 Irrigation aspiration of cortex. The anterior capsular rim is visible all around.

7 mm biconvex optic one piece PMMA implant was inserted into the capsular bag (Fig 9). The fixation was confirmed by visualising the intact circular anterior capsular rim in front of the implant optic (Fig 10). The section was closed with $10 / 0$ nylon suture after aspirating the viscoelastic.

\section{Results}

The preoperative visual acuity of all cases is

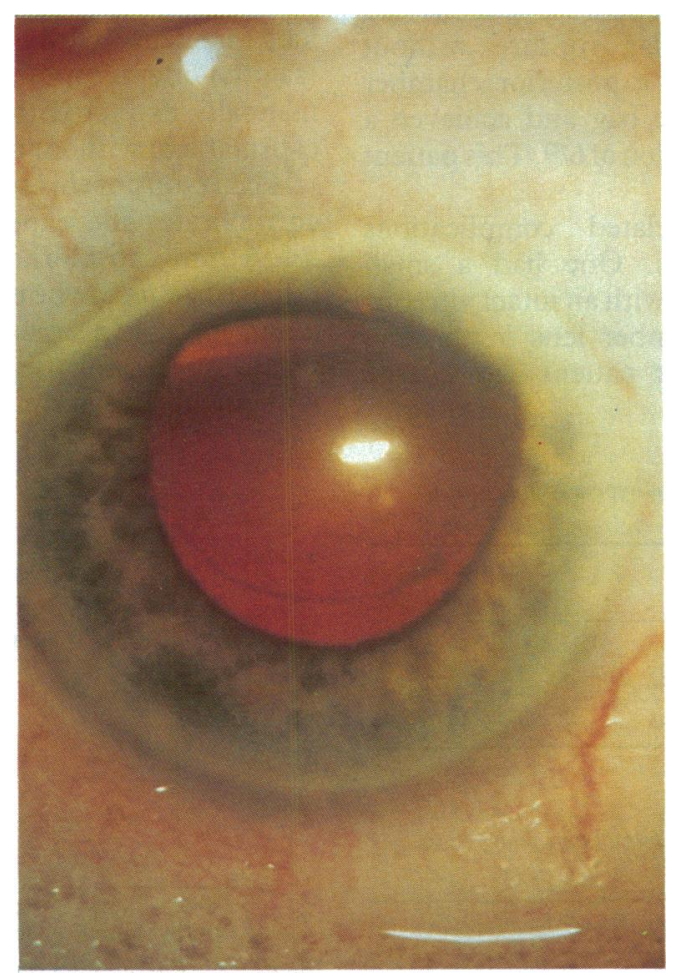

Figure 9 Intraocular lens insertion. The $7 \mathrm{~mm}$ optic stretching the capsular rim is clearly evident.

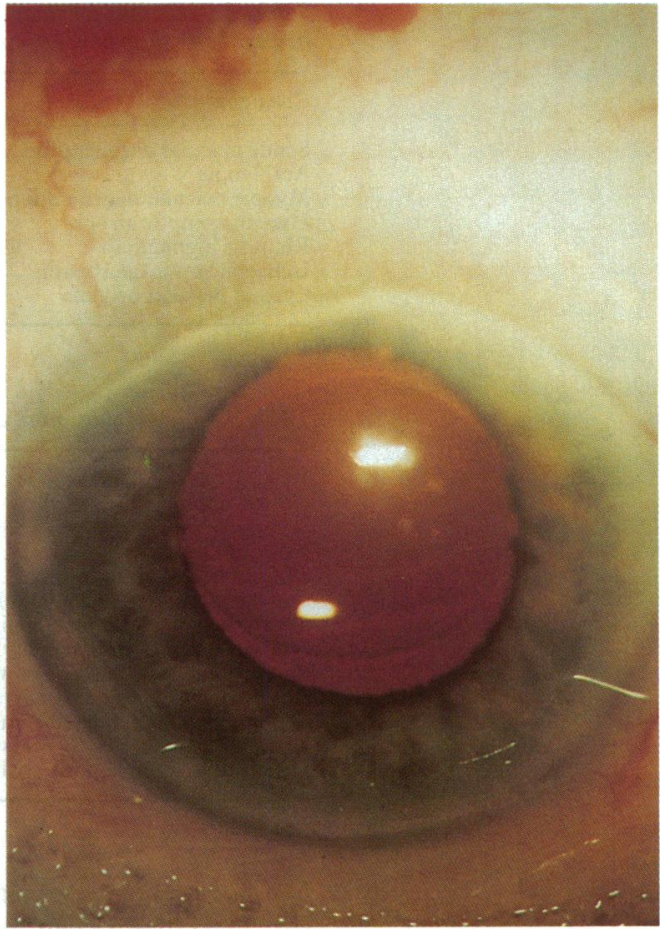

Figure 10 Total bag fixation of intraocular lens. The anterior capsular rim is visible in front of the optic all around.

shown in Table 1. The wide range of preoperative visual acuities and the large percentage of patients with vision of finger counting (39\%) and hand movements (18\%) indicates the representative nature of the sample as regards the density of cataracts.

A final corrected postoperative visual acuity of $6 / 12$ or better was achieved by $81.5 \%$ of all patients. If patients with senile maculopathy are excluded, $93.5 \%$ of patients achieved a final corrected visual acuity of $6 / 12$ or better. Table 2 shows the reasons for a final corrected postoperative visual acuity worse than $6 / 12$ achieved by $18.5 \%$ of all patients. None of these reasons could be attributed directly to the technique used.

\section{COMPLICATIONS}

The incidence of complications in all cases is shown in Table 3: $75 \cdot 23 \%$ of patients had uncomplicated procedures. The complications that occurred were classified as at their time of occurrence in the cataract procedure.

CCC related complications occurred in $22.85 \%$ of patients. Two complications occurred with CCC. In $8.57 \%$ of patients CCC had to be abandoned owing to peripheral extension during capsule tearing (the capsulotomy was converted

Table 1 Best corrected preoperative visual acuity in all cases $(n=210)$

\begin{tabular}{lc}
\hline Visual acuity & Number of cases (\%) \\
\hline $6 / 18$ & $8(4)$ \\
$6 / 24$ & $13(6)$ \\
$6 / 36$ & $33(16)$ \\
$6 / 60$ & $19(9)$ \\
$6 / 60$ - finger counting & $2(1)$ \\
Finger counting & $82(39)$ \\
Hand movements & $38(18)$ \\
Perception of light & $15(7)$ \\
\hline
\end{tabular}


Table 2 Reasons for final corrected postoperative visual acuity worse than $6 / 12(n=39)$

\begin{tabular}{lc}
\hline Condition & Number of cases \\
\hline Senile macular degeneration & 26 \\
Amblyopia & 7 \\
Myopic macular degeneration & 2 \\
Hereditary optic atrophy & 1 \\
Retinitis pigmentosa & 1 \\
Ischaemic optic neuropathy & 1 \\
Cystoid macular oedema & 1 \\
\hline
\end{tabular}

Table 3 Complications in all cases $(n=210)$

\begin{tabular}{lc}
\hline Complication & $\begin{array}{c}\text { Number of } \\
\text { cases }(\%)\end{array}$ \\
\hline No complication & $158(75 \cdot 23)$ \\
CCC related complications & $48(22 \cdot 85)$ \\
Conversion to canopener capsulotomy & $18(8 \cdot 57)$ \\
Micro rim tears (at starting point of CCC) & $30(14 \cdot 28)$ \\
Nucleus delivery related complications & $2(0 \cdot 95)$ \\
Posterior capsular rupture no vitreous (MSC) & $1(0 \cdot 47)$ \\
Accidental intracapsular expression no vitreous & $1(0 \cdot 47)$ \\
Cortical aspiration related complications & $2(0.95)$ \\
Posterior capsular rupture no vitreous & $1(0 \cdot 47)$ \\
Posterior capsular rupture with vitreous & $1(0 \cdot 47)$ \\
\hline
\end{tabular}

to a canopener type). In $14 \cdot 28 \%$ of patients micro rim tears were seen at the time of CCC. Micro rim tears were defined as minute tears in the capsulorhexis rim at the junction of the initial radial and circumferential cuts made in the capsule to raise a capsular flap for tearing. These were seen in cases where the end point of the capsulorhexis coincided with the starting point and the capsulorhexis did not end beyond the starting point in a spiral fashion. These would tear out to the periphery at the time of nucleus delivery.

Nucleus delivery related complications were seen in only two patients. In one patient accidental intracapsular extraction occurred, the vitreous face remained intact and this patient had an anterior chamber lens implanted and attained a corrected vision of $6 / 6$. The other patient had a small posterior capsular rupture without vitreous presentation and a posterior chamber lens was implanted in the bag and achieved a postoperative corrected vision of $6 / 9$. This patient had a mature cataract.

Cortical aspiration related complications occurred in two patients. One had a small posterior capsular rupture with an intact vitreous face and a posterior chamber lens in the bag was implanted. The other patient had a large

Table 4 Complications in mature/hypermature cataract cases $(n=28)$

\begin{tabular}{lc}
\hline Complication & $\begin{array}{c}\text { Number of } \\
\text { cases }(\%)\end{array}$ \\
\hline No complication & $15(53 \cdot 57)$ \\
Conversion to canopener capsulotomy & $8(28 \cdot 57)$ \\
Micro rim tears (at starting point of CCC) & $4(14 \cdot 28)$ \\
Posterior capsular rupture no vitreous & $1(3 \cdot 57)$ \\
\hline
\end{tabular}

Table 5 Complications in small pupil (synechiae) cases $(n=10)$

Complication

Number of cases $(\%)$

No complication

$8(80 \cdot 0)$

Micro rim tears (at starting point ot CCC) posterior capsular rupture with vitreous prolapsing into the anterior chamber, an anterior chamber lens was implanted after an anterior vitrectomy. Both patients attained a corrected vision of $6 / 9$ postoperatively.

The complications in mature/hypermature cataracts and small pupil (pupillary synechiae) cases are shown in Tables 4 and 5 respectively. The relative increase in CCC related complications reflects the difficulty in tearing the capsule in these cases due to poor visualisation of the anterior capsule.

The complications were also analysed with respect to their incidence over time. The learning curve involved in CCC is apparent from Fig 11. The two cases in which nucleus delivery related complications occurred were operated on during the first 6 months of the technique being used. Since then more than 140 cases were operated on without any nucleus delivery related complications.

\section{Discussion}

Various authors ${ }^{2-6}$ have suggested that radial relieving rim cuts are necessary for safe nucleus delivery when using CCC in ECCE. This however defeats the primary purpose of achieving a completely intact capsular bag. ${ }^{7}$ Witteman ${ }^{8}$ suggested the use of a large 7-8 $\mathrm{mm}$ capsulorhexis for nucleus expression. It is technically difficult to produce such a large opening and disrupts the zonular insertion in the anterior capsule. The capsule does not tear smoothly as it repeatedly comes across the insertion of the zonular fibres.

Experimental human cadaver eye studies ${ }^{910}$ clearly demonstrate the ability of the anterior capsular rim to stretch well beyond the dimensions required for safe nucleus delivery. Assia et $a l^{10}$ demonstrated that the capsular rim in CCC can be stretched to 1.6 times its original circumference before tearing. They calculated that a lens nucleus with a profile circumference (sagittal or anteroposterior) of 18-22 $\mathrm{mm}$ can be manually extracted through a $5.5 \mathrm{~mm}$ anterior capsular rim without tearing.

The results of this series of 210 cases clinically corroborates the experimental evidence that a 5.5-6 mm capsulorhexis opening without any radial rim cuts can be used successfully in ECCE. Thim et $a l,{ }^{11}$ in a series of 26 cases, also reported on the successful use of a $6 \mathrm{~mm}$ capsulorhexis without any radial rim cuts.

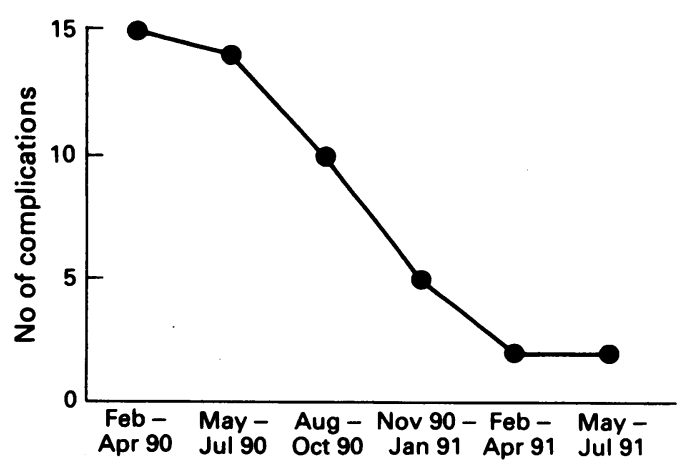

Figure 11 The learning curve. The frequency of complications decrease over time. The number of operations in each 3 month period was roughly the same. 
Nucleus delivery related complications occurred in only two of 210 cases. These occurred in the first 6 months of using this technique. Since then 140 cases have been operated on without any nucleus delivery related complications. Accidental intracapsular lens expression occurred in one patient, while the other patient (mature cataract) had a posterior capsular rupture at nucleus delivery. Both these patients had final visual acuities of better than $6 / 12$. Thim et $a l^{\prime 1}$ did not report any nucleus delivery related complications in their small series of 26 patients using a similar technique for nucleus delivery.

Harris and Specht ${ }^{2}$ reported six cases of intracapsular lens delivery from five different surgeons at three different institutions on attempted nuclear expression after CCC. They surmise that this is a relatively common complication. Various authors ${ }^{2-4}$ have also reported similar accidental intracapsular lens expression. Conventional nuclear expression techniques which did not involve a global hydrodissection of the nucleus before tilting and expression were used in most of these reported cases.

The low rate of nucleus delivery related complications in the current study reflects the different nucleus delivery technique used. The basic principles of this technique are: (1) global hydrodissection to completely free the nucleus of its cortical attachment so that it can be manoeuvred independently of the cortex and the capsular bag; (2) delivery of the nuclear pole through the capsular rim. This requires tilting the nucleus to prolapse the nuclear pole through the capsular rim. This tilt can be achieved by injecting irrigating fluid in the capsular bag or by differential limbal pressure; (3) rotation of the prolapsed nuclear pole into the section and expression. Shimuzu ${ }^{12}$ and Corydon and Thim ${ }^{13}$ described techniques for nuclear delivery when using CCC in ECCE based on similar principles.

Posterior capsular-zonular disruption occurred in only four of the 210 cases $(1 \cdot 88 \%)$. This compares well with the $4 \cdot 8-7 \cdot 9 \%$ reported rates of posterior capsular-zonular disruption in planned ECCE using canopener/linear capsul${\text { otomies. }{ }^{14} \text { is }}$

The mechanics of safe nuclear delivery requires that the size of the capsular opening should be large enough to allow nuclear egress by applying a force smaller than that of zonular rupture. The nucleus delivery technique used in this study seems to meet this criterion.

CCC-related complications occurred in $23 \%$ of the patients. In $8 \cdot 57 \%$ of all patients peripheral extension of the capsulorhexis necessitated converting the capsulotomy to a canopener capsulotomy. The conversion to canopener capsulotomy rate was higher $(28.57 \%)$ in cases with mature or hypermature cataracts. This reflects the technical difficulty in controlled tearing of the capsule in the absence of a good red reflex. Micro rim tears at the starting point of the capsulorhexis were seen in $14 \cdot 28 \%$ of patients. These small tears extended to the periphery when the nucleus was delivered. These tears were avoided when the (capsulorhexis) tearing was continued beyond the starting point of the capsulorhexis - in effect a spiral tearing of the capsule. Micro rim tears were also more common in cases with small pupils or mature cataracts reflecting further the technical difficulties in achieving an optimal CCC.

The number of complications decreased consistently with time. As most of the complications were related to capsulorhexis the learning curve is related to the technique of CCC.

\section{Conclusion}

The success of this technique is apparent from the low incidence of complications seen in this large series. The author believes that by using this technique the well established advantages of CCC can be of benefit to patients having planned ECCE. Further CCC should be the capsulotomy technique of choice with ECCE.

Part of this paper was presented as a poster at the Annual conference of the United Kingdom Intraocular Implant Society October 1991

I thank Mrs U K Goddard, Mr A K Mathur, Mr S K Datta, and Mr J R Innes for their encouragement and permission to use patients under their care for this study.

1 Gimbel HV, Neuhann T. Development, advantages and methods of the continuous circular capsulorhexis technique. f Cataract Refract Surg 1991; 16: 31-7.

2 Harris DJ, Specht CS. Intracapsular lens delivery during attempted extracapsular cataract extraction, association with capsulorhexis. Ophthalmology 1991; 98: 623-7.

3 Alamallah OF. Capsulorhexis complications with planned extracapsular cataract extraction [Letter]. F Cataract Refract Surg 1989; 15: 232-3.

4 Hunyor ABL. Avoiding capsulorhexis complications with extracapsular cataract surgery [Letter]. $\mathcal{F}$ Cataract Refract Surg 1989; 15: 464 .

5 Maher J. Nucleus expression after capsulorhexis [Letter]. f Cararact Refract Surg 1988; 14: 693 .

6 Lee CP. Capsulorhexis - a 5 year experience. Eur 7 Implant Refract Surg 1990; 2: 27-31.

7 Blumenthal M, Assia E, Neumann D. The round capsulorhexis capsulotomy and the rationale for $11.0 \mathrm{~mm}$ diameter IOL. Eur F Implant Refract Surg 1990; 2: 15-9.

8 Witteman GJ. Avoiding capsulorhexis complications with extracapsular cataract surgery [Letter]. $\mathcal{F}$ Cataract Refract Surg 1989; 15: 463-4.

9 Thim K, Krag S, Corydon L. Stretching capacity of capsulorhexis and nucleus delivery. $\mathcal{F}$ Cataract Refract Surg 1991; 17: 27-31.

10 Assia EI, Apple DJ, Tsai JC, Lim ES. The elastic properties of the lens capsule in capsulorhexis. Am F Ophthalmol 1991; 111: $628-32$.

11 Thim K, Krag S, Corydon L. Capsulorhexis and nucleus expression. Eur f Implant Refract Surg 1990; 2: 37-41.

12 Shimuzu K. Double hook extraction technique. F Cataract Refract Surg 1989; 15: 702-4.

13 Corydon L, Thim K. Continuous circular capsulorhexis and nucleus delivery in planned extracapsular cataract extraction. F Cataract Refract Surg 1991; 17: 628-32.

14 O'Donnell FE, Santos B. Posterior capsular-zonular disruption in planned extracapsular surgery. Arch Ophthalmol 1985; 103: 652-3.

$15 \mathrm{Nishi} \mathrm{O}$. Vitreous loss in posterior chamber lens implantation. f Cataract Refract Surg 1987; 13: 424-7. 